\title{
Study of a possible scintillating fibre tracker at the LHC and tests of scintillating fibres
}

\author{
D. Autiero, P. Camarri, A. Cardini, V. Cavasinni, T. Del Prete, B. Di Girolamo, V. Flaminio and \\ E. Iacopini \\ Dipartimento di Fisıca, Unıversità di Pisa and Istituto Nazionale dı Fisica Nucleare, Sezlone di Pisa, Italy
}

\author{
A. Gorin and I. Manuilov \\ IHEP, Protvino, Russian Federation
}

Received 2 April 1993 and in revised form 2 July 1993

\begin{abstract}
We present a possible scheme for a scintillating fibre tracker that could be used at the LHC in the central pseudorapidity region. The experimental environment at the LHC was simulated in detail by a Monte Carlo program using the GEANT package. We shall discuss the expected performance of the proposed tracker, mainly for electron identification. We also report on the results of laboratory measurements of scintillating fibre characteristics: we measured the time response, the light yield, the attenuation length and the light propagation speed in individual fibres, lookıng for the best candidates for the LHC.
\end{abstract}

\section{Introduction}

The experiments which will be performed at future high energy and high luminosity hadronic colliders (LHC, SSC) will face severe experimental problems: the most remarkable ones are a high proton-proton collision rate and a high radiation level [1]. In particular a tracking detector operating in the central region $(|\eta|<2)$ should be:

- radiation resistant ( $\sim 1 \mathrm{Mrad})$;

- accurate: for momentum and charge measurement up to $\sim 1 \mathrm{TeV}$; tion;

- granular enough to limit fake track reconstruc-

- fast: it should provide signals every bunchcrossing (15 ns);

- transparent to electromagnetic radiation, i.e. total thickness $\lesssim 0.1$ r.l.

We studied a central tracker made of scintillating fibers; its main aim is to contribute to identify (at the first level trigger) events with high $p_{\mathrm{T}}$ leptons originating in a pp collision - as well as to measure the lepton momentum - out of the $\sim 10^{9}$ events per second occurring at the LHC (pp collisions at $16 \mathrm{TeV}$ ) at the highest luminosity $\left(10^{34} \mathrm{~cm}^{-2} \mathrm{~s}^{-1}\right)$.

\section{Monte Carlo simulation of the scintillating fibre tracker}

We propose a scintillating fibre ( $\mathrm{SciFi}$, diameter $=0.5 \mathrm{~mm}$ ) tracking device aiming to fulfil the above mentioned requirements, i.e.:

- high radiation hardness;

- very fast time response;

- good space resolution;

- good transparency.

The tracker performances at the LHC were studied by a detailed Monte Carlo program providing a full simulation using the GEANT package [2]. The geometry of the SciFi tracker is the following (see fig. 1):

- The fibres (each one with a diameter of $0.5 \mathrm{~mm}$ ) positioned along the beam axis (to provide $r, \phi$ coordinates) are grouped into cylindrical "superlayers": a superlayer is made of two series of four fibre layers each, with a spacing of $4.6 \mathrm{~cm}$ (the overall thickness of a superlayer is, therefore, $5 \mathrm{~cm}$ ); a staggering of $125 \mu \mathrm{m}$ ( $1 / 4$ of the fibre diameter) between contiguous layers gives a space resolution $r \Delta \phi \approx 40 \mu \mathrm{m}$ at full efficiency (fig. 1). The simulation includes also a beryllium pipe and suitable carbon fibre supports for fibres.

- A first SciFi superlayer is positioned $0.5 \mathrm{~m}$ and another one $1 \mathrm{~m}$ from the beam axis, with acceptance $|\eta|<1.9$ (fig. 2); besides, the fibre layers are interrupted at $\eta=0$ to separate left/right signals.

The detector includes a total of 281600 fibres and the total thickness at $\eta=0$ is $\sim 5 \%$ r.l. The light sig- 


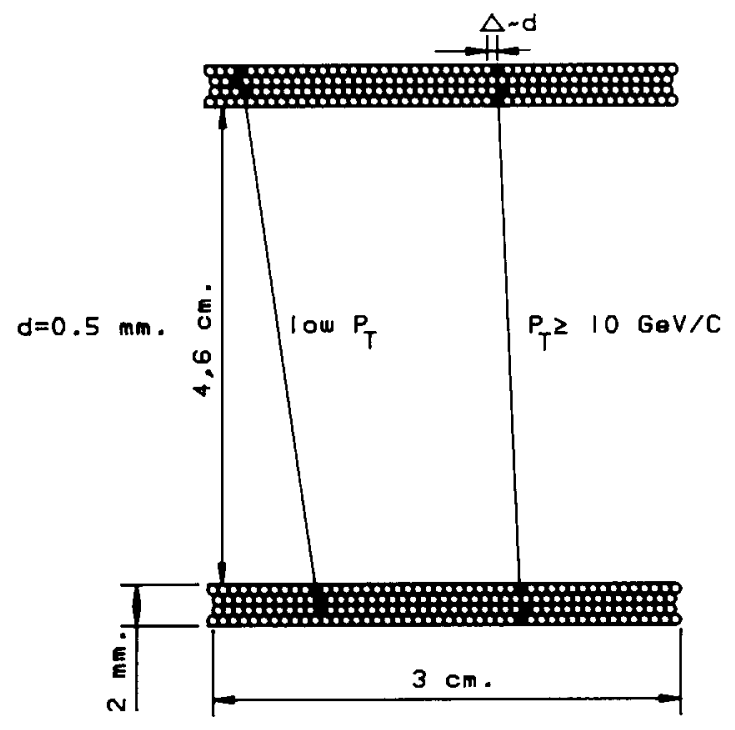

Fig. 1. Transverse section of a superlayer of the proposed scintillating fibre detector; possible hits for low $p_{\mathrm{T}}$ and high $p_{\mathrm{T}}$ charged particles are represented.

nal is brought far from the central region of the detector, so that the readout electronics are not affected by the high radiation dose; for this reason, in the pro- posed scheme, each scintillating fibre is prolonged by using an $8 \mathrm{~m}$ long clear light guide. The price to pay is a low average number of photons arriving at the far end of the clear fibre; thus, the readout must be performed through high efficiency photon detectors. We assume to use the VLPCs (Visible Light Photon Counters, produced by Rockwell), which have quantum efficiency $\sim 85 \%$ in the visible region of the electromagnetic spectrum [3].

The simulation includes trapping efficiency, attenuation effects $(2.5 \mathrm{~m}$ in the scintillating fibres, $12 \mathrm{~m}$ in the clear fibres) and efficiency factors of clear/scintillating fibre junctions and VLPC.

The Monte Carlo calculation was performed with three kinds of events (generated in pp collisions at 16 TeV):

- isolated electrons with energy $>20 \mathrm{GeV}$;

- QCD jets $\left(E_{J}>20 \mathrm{GeV}\right)$ which fake electromagnetic clusters in the calorimeter; at the calorimeter level the electromagnetic cluster was selected by:
a) $|\eta|<2$;
b) $E_{\text {em }}(\Delta \eta \times \Delta \phi=0.06 \times 0.06)>20 \mathrm{GeV}$,
c) $E_{\text {leak }}(\Delta \eta \times \Delta \phi=0.06 \times 0.06)<5 \mathrm{GeV}$,
d) $E_{\text {sol }}(\Delta \eta \times \Delta \phi=0.1 \times 0.1)<10 \mathrm{GeV}$,

providing already a rejection factor $\simeq 240$ against QCD jets;

- minimum bias events.

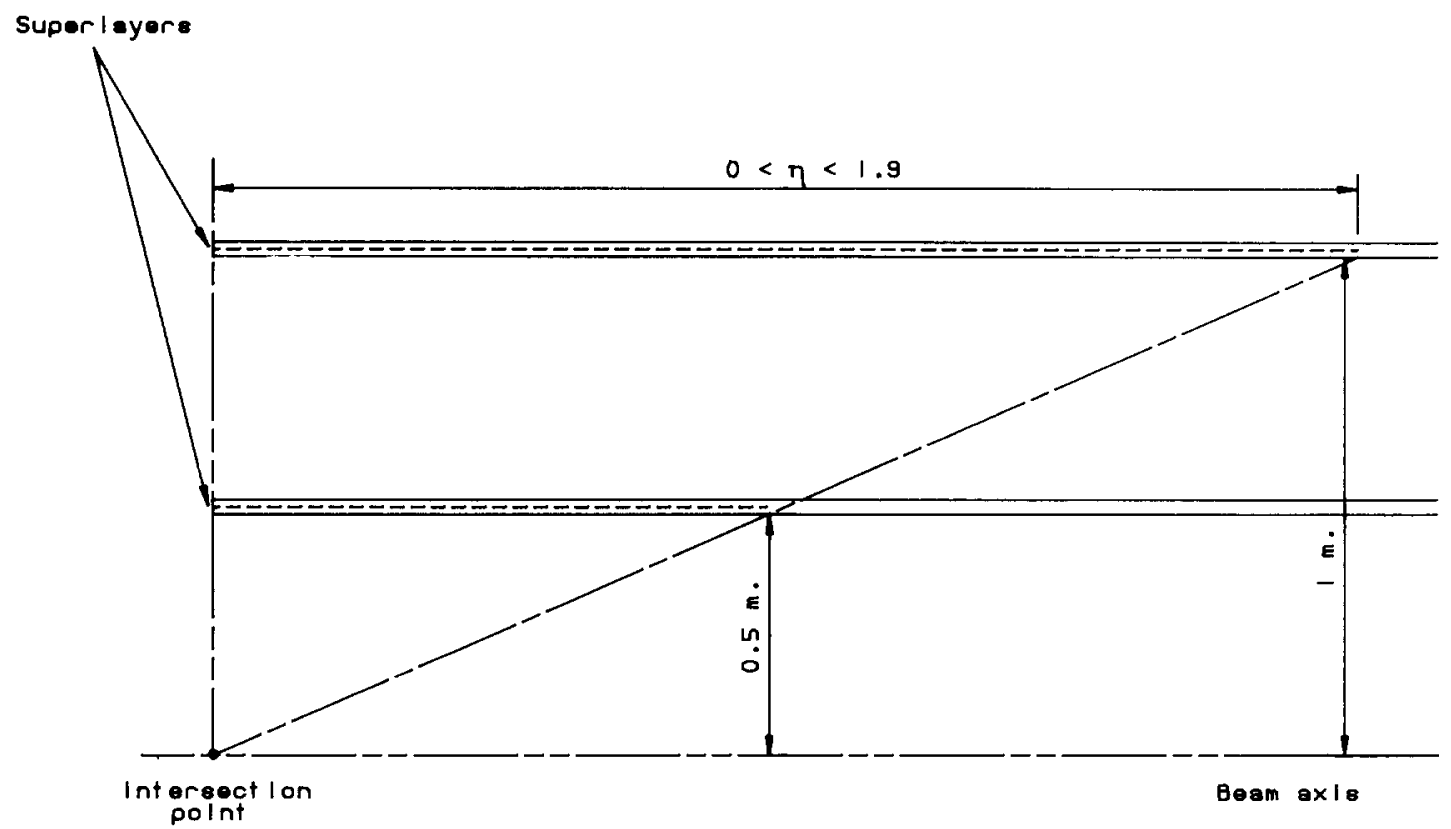

Fig. 2. Longitudinal section of the proposed detector; in this scheme only $1 / 4$ of the whole section is represented. 

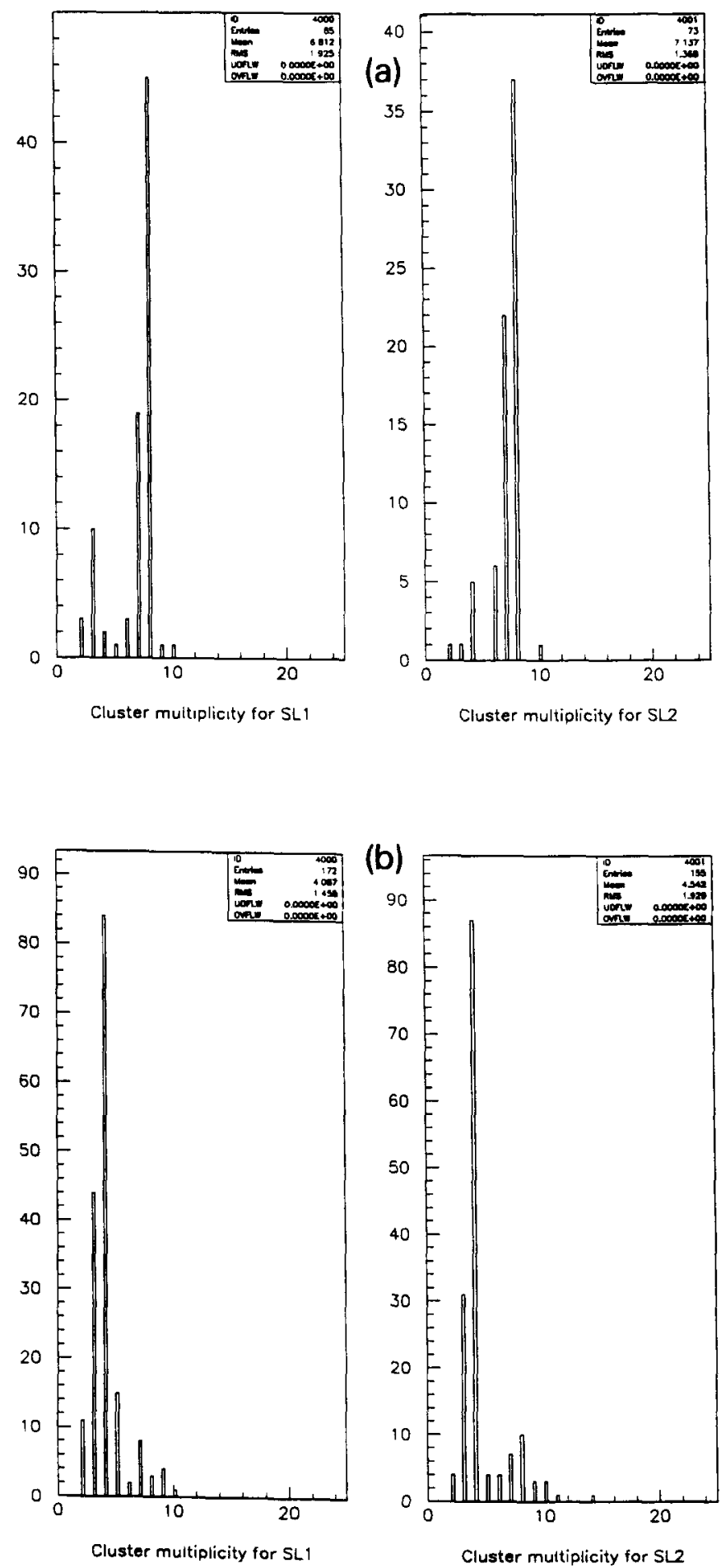

Fig. 3. (a) Distribution of cluster multiplicities in superlayers 1 and 2 for electron tracks at low luminosity. (b) Distribution of cluster multiplicities in superlayers 1 and 2 for QCD jet tracks at low luminosity. 
An "electron" is a charged track with reconstructed momentum matching the calorimeter energy and pointing to the electromagnetic cluster of the calorimeter within an angular fiducial region.

In order to simulate the high LHC luminosity, we superimpose minimum bias events on both electron and QCD jet events: at $\mathcal{L}=10^{33} \mathrm{~cm}^{-2} \mathrm{~s}^{-1}$ the average rate is 1 minimum bias event per bunch crossing (rate $\sim 10^{8} \mathrm{~s}^{-1}$ ); at $\mathcal{L}=10^{34} \mathrm{~cm}^{-2} \mathrm{~s}^{-1}$ the corresponding rate is 10 minimum bias events per bunch crossing (rate $\sim 10^{9} \mathrm{~s}^{-1}$ ).

By applying a solenoidal magnetic field, it is possible to reconstruct the transverse momentum of the charged tracks; in a $2 \mathrm{~T}$ magnetic field, the transverse momentum $p_{\mathrm{T}}$ and the bending radius $\rho$ of a track are linked through the following dependence:

$p_{\mathrm{T}}(\mathrm{Ge} V / \mathrm{c})=0.6 p(\mathrm{~m})$.

The $p_{\mathrm{T}}$ resolution resulting from the proposed detector geometry and the applied magnetic field is:

$\frac{\delta p_{\mathrm{T}}}{p_{\mathrm{T}}} \approx 0.5 p_{\mathrm{T}}(\mathrm{TeV} / \mathrm{c})$.

The number of charged particles coming from a minimum bias event at the LHC (per unit pseudorapidity) is [4]:

$\frac{d n_{\mathrm{ch}}}{d \eta} \simeq 6$.

Then, we estimate that about 230 charged particles per bunch-crossing will emerge from the interaction region at $\mathcal{L}=10^{34} \mathrm{~cm}^{-2} \mathrm{~s}^{-1}$.

In our simulation of the LHC running conditions we neither did include the effect of back-splash particles from the material surrounding the cavity of the central detector nor the signals produced by neutrons originated by the outer calorimeters. These effects depend on the configuration of the outer detectors and they could increase the noise in the fibre tracker. In the ATLAS experiment at the LHC [5] the amount of back-splash from the cryostat of the magnet coil surrounding the central region was estimated not to exceed $10 \%$ of the total number of the minimum bias particles. In this experiment the neutron albedo was also calculated: at the maximum LHC luminosity the neutron fluence per year is $10^{13} \mathrm{n} / \mathrm{cm}^{2}$ resulting in about 200 neutrons crossing a layer of the fibre tracker every $15 \mathrm{~ns}$. We estimate an additional number of about 20 hits in a layer of fibres and this is still negligible compared to the hits due to the primary particles.

In our detector a stiff track $\left(p_{\mathrm{T}}>10 \mathrm{GeV} / c\right)$ is recognized because its hits in a superlayer ( 8 at full efficiency) are lined up within one fibre diameter. Due to the high particle flux, random correlation of hits coming from minimum bias tracks could also mimic a large $p_{\mathrm{T}}$ electron: we calculated this contamination to be $<1 \%$, provided we know the $\phi$ cone of the electron $\left( \pm 2.5^{\circ}\right)$ defined by the calorimeter cluster position.

In the Monte Carlo, high $p_{\mathrm{T}}$ tracks are selected using the following algorithm:

- "hits" are searched for within $\pm 2.5^{\circ}$ around the electron or jet direction (set by the calorimeter spatial resolution ): the average number of photoelectrons measured for each fibre is about 10 ;

- for each superlayer, $\geq 7$ fibres lined up with the
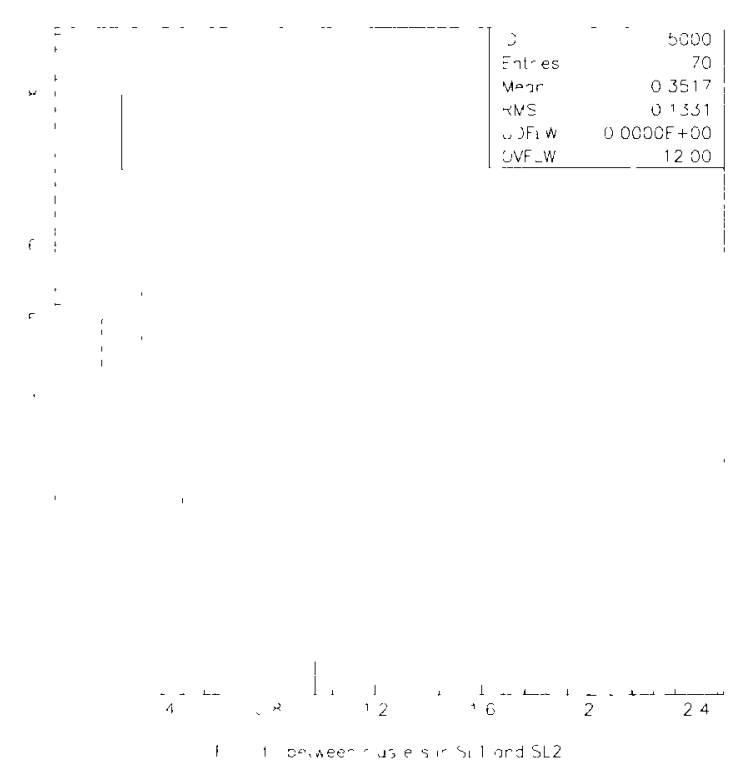

Fig. 4. $\Delta \phi$ distribution for electron tracks at low luminosity.

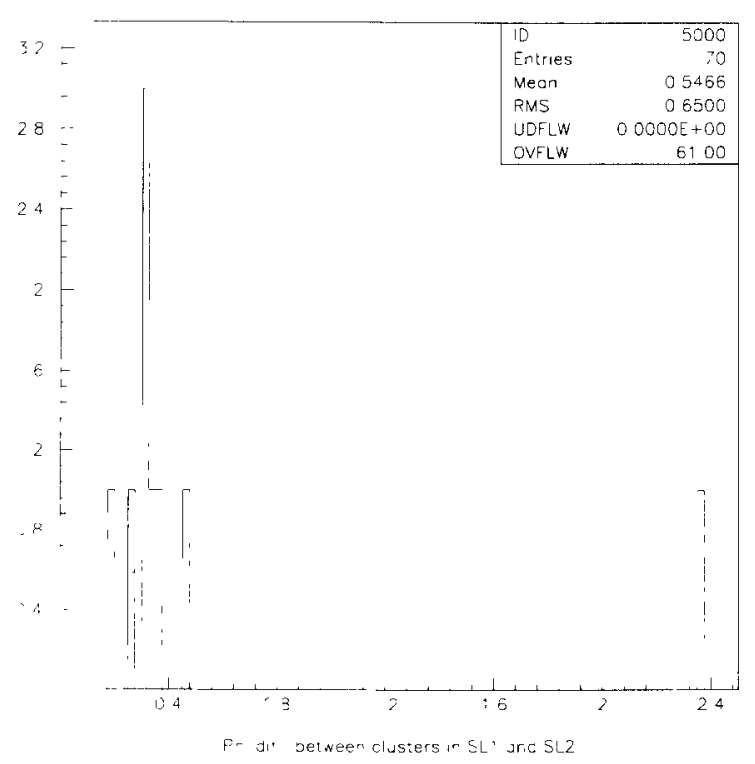

Fig. 5. $\Delta \phi$ distribution for QCD jet tracks at low luminosity. 

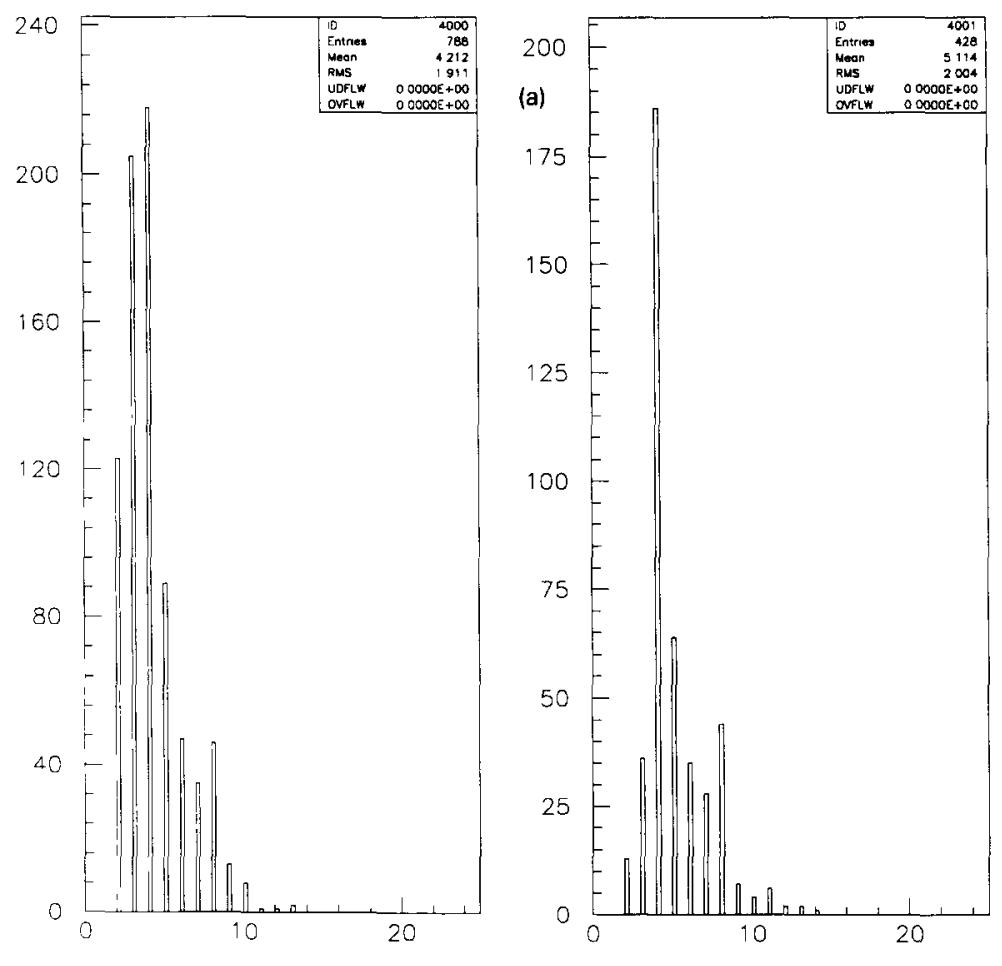

Cluster multiplicity for SL1
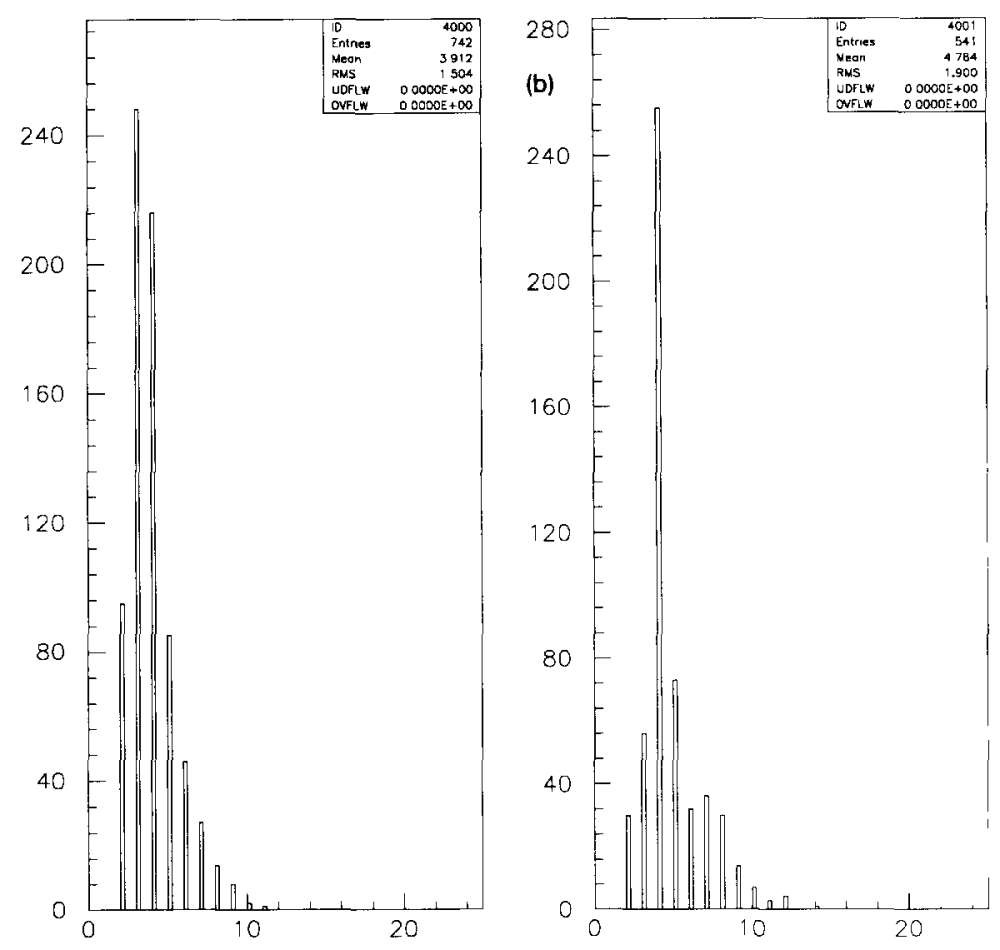

Cluster multiplicity for SL 1

Cluster muitiplicity for SL2

Fig. 6. (a) Distribution of cluster multiplicities in superlayers 1 and 2 for electron tracks at high luminosity. (b) Distribution of cluster multiplicities in superlayers 1 and 2 for QCD jet tracks at high luminosity. 


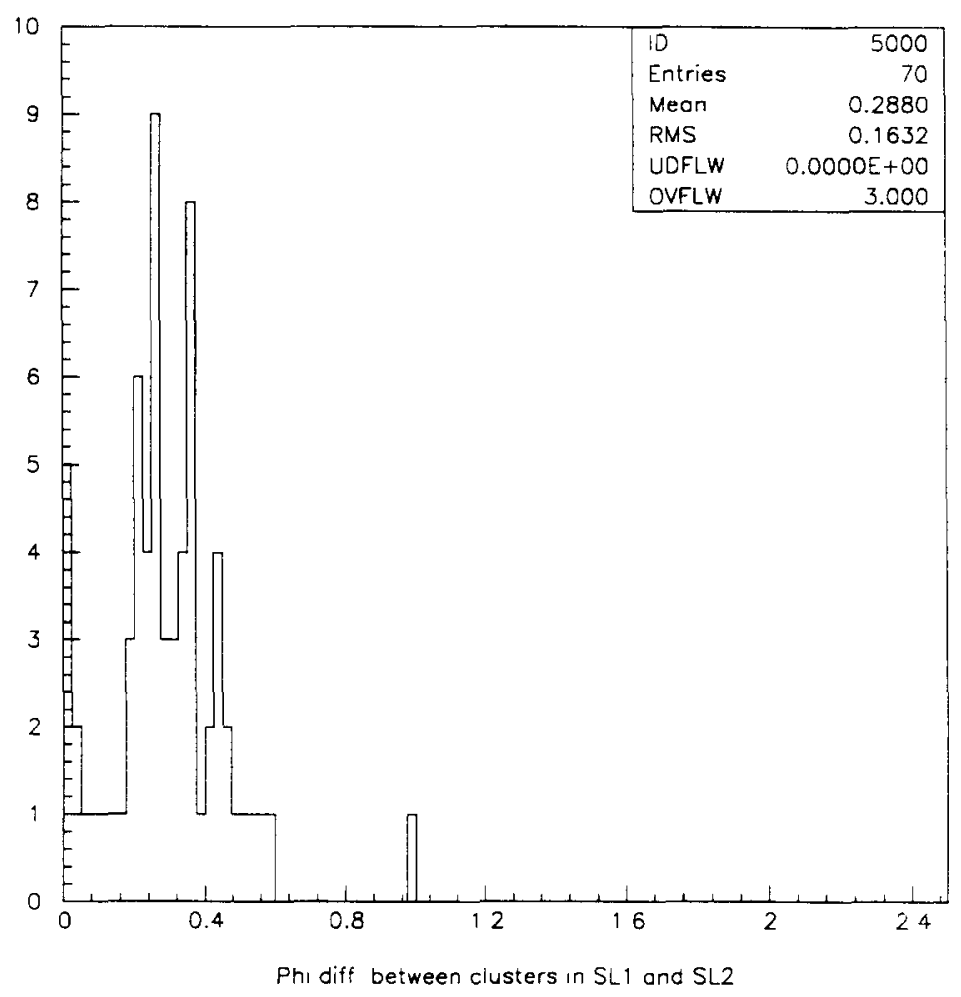

Fig. 7. $\Delta \phi$ distribution for electron tracks at high luminosity.

interaction vertex within one fibre diameter $\left(p_{T}>10\right.$ $\mathrm{GeV})$ are selected; average angular coordinates $\left(\left\langle\phi_{1}\right\rangle\right.$ in the inner superlayer, $\left\langle\phi_{2}\right\rangle$ in the outer one) are assigned to these "clusters";

- finally, we study the histogram of the quantity

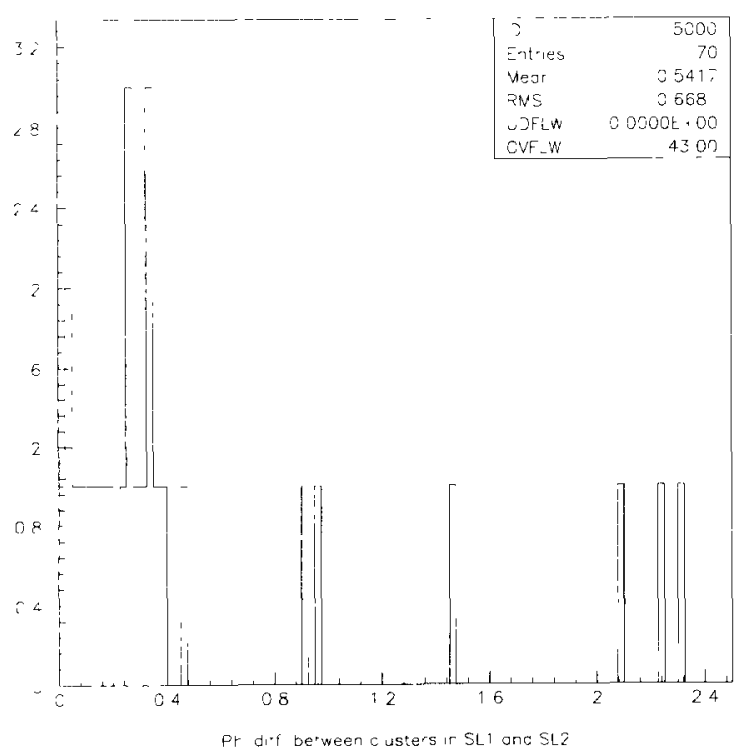

Fig. 8. $\Delta \phi$ distribution for QCD jet tracks at high luminosity. $\left|\left\langle\phi_{1}\right\rangle-\left\langle\phi_{2}\right\rangle\right|:$ if there is more than one possible correlation between clusters in the two superlayers, we consider the pair of clusters for which $|\Delta \phi|$ is minimum.

This procedure was used first to analyse electron events and QCD jet events, with one minimum bias event overlapped (low luminosity). For the electrons (fig. 3a), we see that the fibre multiplicity of most tracks in each superlayer equals 8 (it corresponds to a track crossing one fibre in each layer); from the $|\Delta \phi|$ distribution (fig. 4), a clear correlation signal is visible: setting a cut at $\Delta \phi=0.8^{\circ}$ we get an electron efficiency of $95 \%$.

For the QCD jets (fig. 3b), however, the average multiplicity of the clusters in each superlayer is only 4 (a jet contains many low energy particles, and their paths do not cross all the layers in a superlayer within one fibre diameter). A similar $\Delta \phi$ cut in jet events retains $\sim 5 \%$, thus the tracker alone provides a rejection factor against jets of $\sim 20$ (fig. 5).

We proceed in an analogous way to analyse events at high luminosity $\left(\sim 10^{34} \mathrm{~cm}^{-2} \mathrm{~s}^{-1}\right)$; now the situation is complicated by the 10 minimum bias events superimposed to a high $p_{\mathrm{T}}$ electron or QCD jet. As shown in fig. 6a (electron events), most clusters (originating from low $p_{\mathrm{T}}$ particles) have low multiplicity and are filtered out by the selection criteria, and again, the electron efficiency at $\Delta \phi<0.8^{\circ}$ is $\sim 95 \%$ (fig. 7). A more difficult situation arises in the reconstruction 


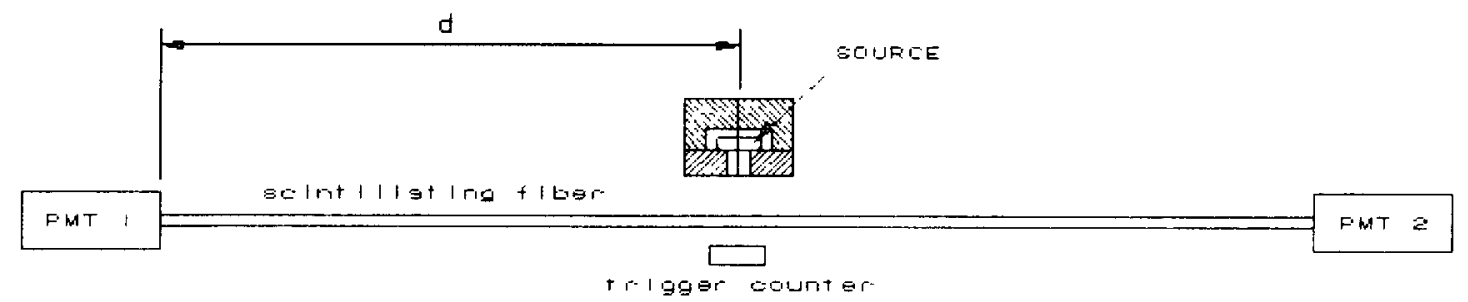

Fig. 9. Longitudinal view of the set-up used in the RIFOS experiment for the measurements of scintillating fibres.

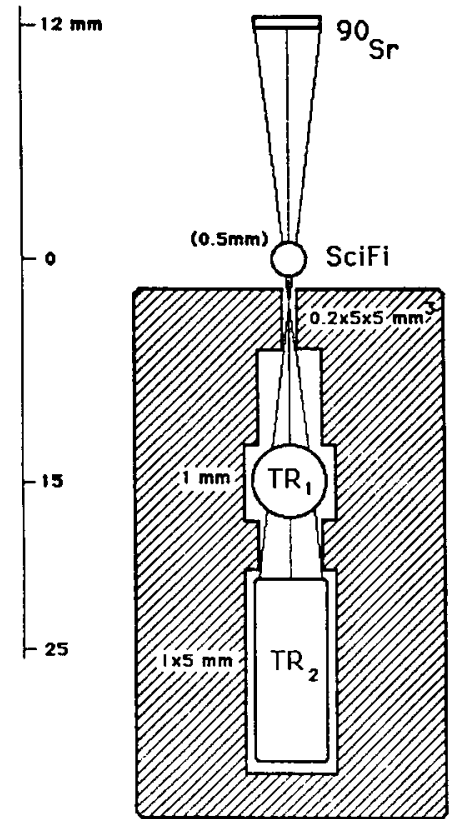

Fig. 10. Scheme of the transverse section of the trigger system used in the RIFOS experiment.

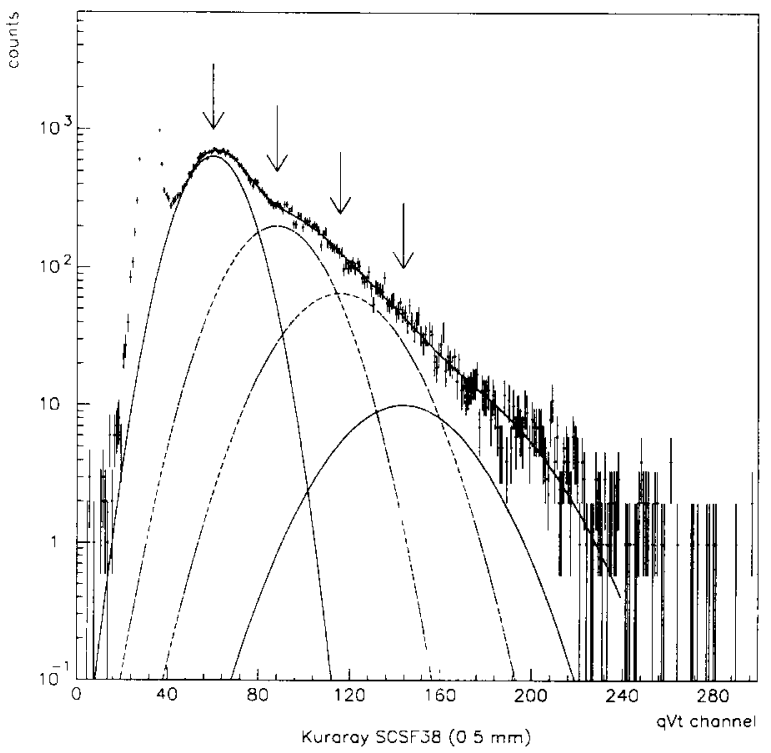

Fig. 11. Example of an experimental $q V t$ charge distribution recorded in the RIFOS experiment; the contributions of 1 , 2, 3 and 4 photoelectrons are shown.

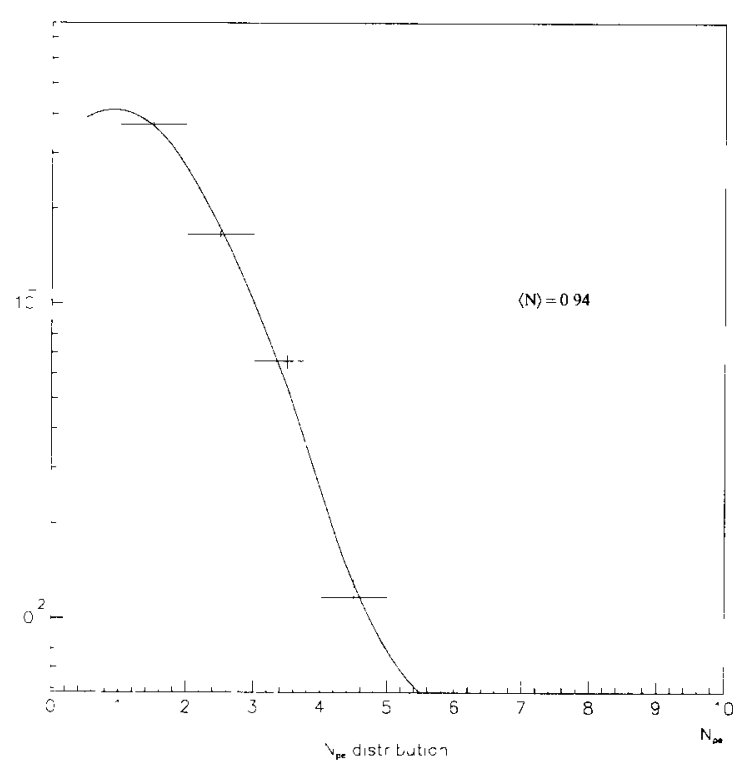

Fig. 12. Poisson distribution fitting the values of the photoelectron peak amplitudes obtained from fig. 11 ; in this case we get 0.94 for the average number of photoelectrons.

of tracks in the jets: the superlayer clusters (fig. 6b) still have low multiplicity, on average, but the probability of random clustering rises due to the large number of clusters and statistical fluctuations: now we find $25 \%$ of jet events with $|\Delta \phi|<0.8^{\circ}$, and the resulting hadron rejection factor is poorer $(\simeq 4$, see fig. 8$)$.

To use the information of high $p_{\mathrm{T}}$ electron tracks for event selection at the first level trigger requires an electron/jet rejection factor of $\sim 10-10^{2}$ provided by the tracker alone [6]. Therefore the proposed scheme is adequate at low luminosity; at high luminosity we estimate that a third superlayer added to the proposed detector will provide the required rejection factor.

We recall the parameters used in our simulation:

- fibre attenuation length (at great distance) = $2.5 \mathrm{~m} ; 12 \mathrm{~m}$ for clear fibres; $95 \%$;

- clear fibre/scintillating fibre junction efficiency:

- photomultiplier quantum efficiency: $85 \%$;

- time interval between two readouts of the same fibre: 15 ns (interval between two bunch-crossings at the LHC).

As a result we find an average number of photons per 


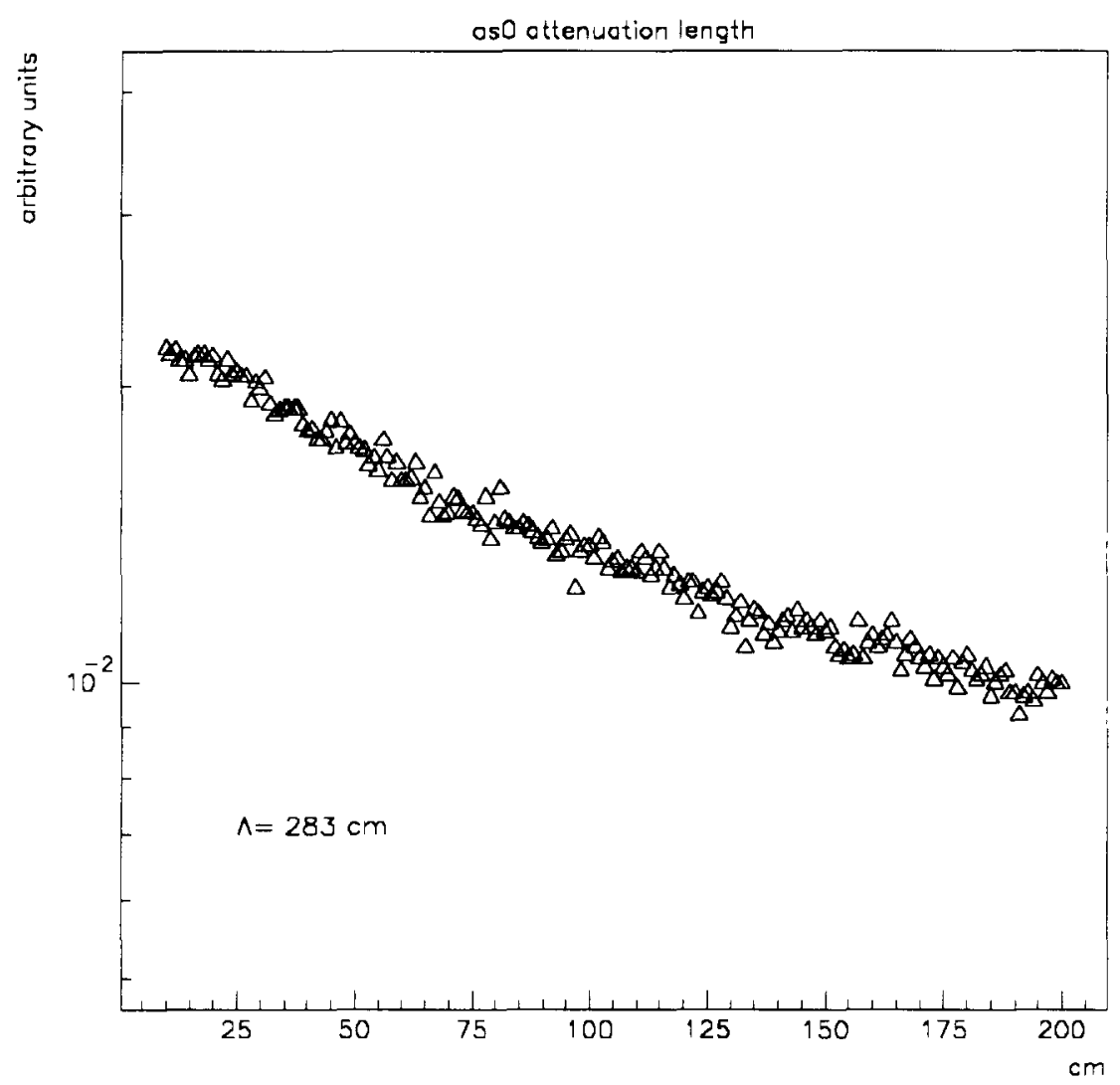

Fig. 13. Example of an experimental attenuation plot of the RIFOS experiment, in this case we get an attenuation length of $283 \mathrm{~cm}$.

fibre (extrapolated to zero distance) $n_{0}=15$. These values have to be kept in mind as a reference for the laboratory tests of scintillating fibres.

\section{Measurements of scintillating fibres}

The RIFOS experiment, performed at the INFN laboratories in Pisa, aims to assess the performance of various kinds of scintillating fibres. The scintillation process is stimulated by a collimated beam of electrons coming from the $\beta^{-}$decay of a $3.7 \mathrm{MBq}{ }^{90} \mathrm{Sr}$ source.

Here are the features of the experimental set-up (fig. 9):

- a $3 \mathrm{~m}$ long fibre is stretched horizontally, $10 \mathrm{~cm}$ above the plane of an optical bench;

- the source is fixed to a small carriage which can be moved all along the fibre on a railway by a stepping motor: a CAMAC module controls the motor movement, and a single step corresponds to a carriage displacement of $0.47 \mathrm{~mm}$;

- the fibre ends are in contact with the input windows of two Hamamatsu R1635-02 photon count- ing photomultipliers, reading the scintillation pulses; the photocathode is bialkali, with quantum efficiency $\simeq 25 \%$ for visible light with $\lambda=420-430 \mathrm{~nm}$, and $\simeq 10 \%$ for $\lambda=530 \mathrm{~nm}$;

- a trigger system is also fixed to the carriage (fig. 10): a collimating slit and two small segments of a scintillating fibre are placed beneath the test fibre; each segment is in contact with a photomultiplier;

- the whole set-up is mounted inside a room which is totally darkened during the measurements.

The following scintillating fibres were measured:

- Pol. Hi. Tech. ${ }^{\# 1}$ fibres $(1 \mathrm{~mm}$ and $0.5 \mathrm{~mm}$ thick) emitting scintillation light at 420,430, 460, $530,590,600$ and $650 \mathrm{~nm}$ (referred to as al for $1 \mathrm{~mm}$ and as for $0.5 \mathrm{~mm}$ diameter).

- Kuraray ${ }^{\# 2}$ fibres $(0.8 \mathrm{~mm}$ thick $)$ emitting scintillation light at $530 \mathrm{~nm}$ (referred to as K3HF).

\#1 Pol. Hi. Tech. s.r.l., S.P. Turanense km 44.400, 67061 Carsoli (AQ), Italy.

\#2 Kuraray Co., Ltd., Methacrylic Resin Division, Shuwa Higash1-Yaesu Building 9-1, Hatchobory 2-Chome, Chuo-ku, Tokyo 104, Japan. 


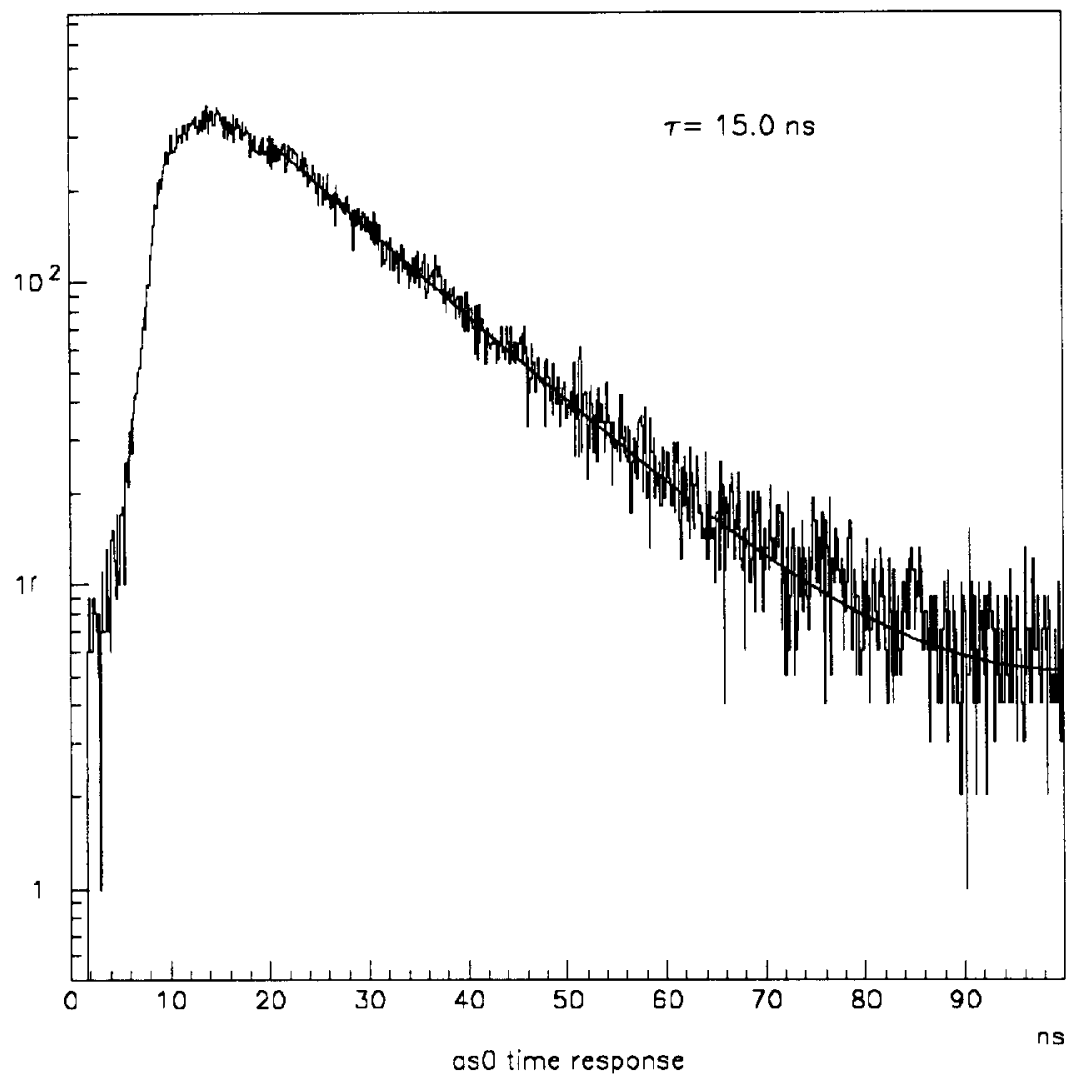

Fig. 14. Example of a time response measurement performed in the RIFOS experiment.

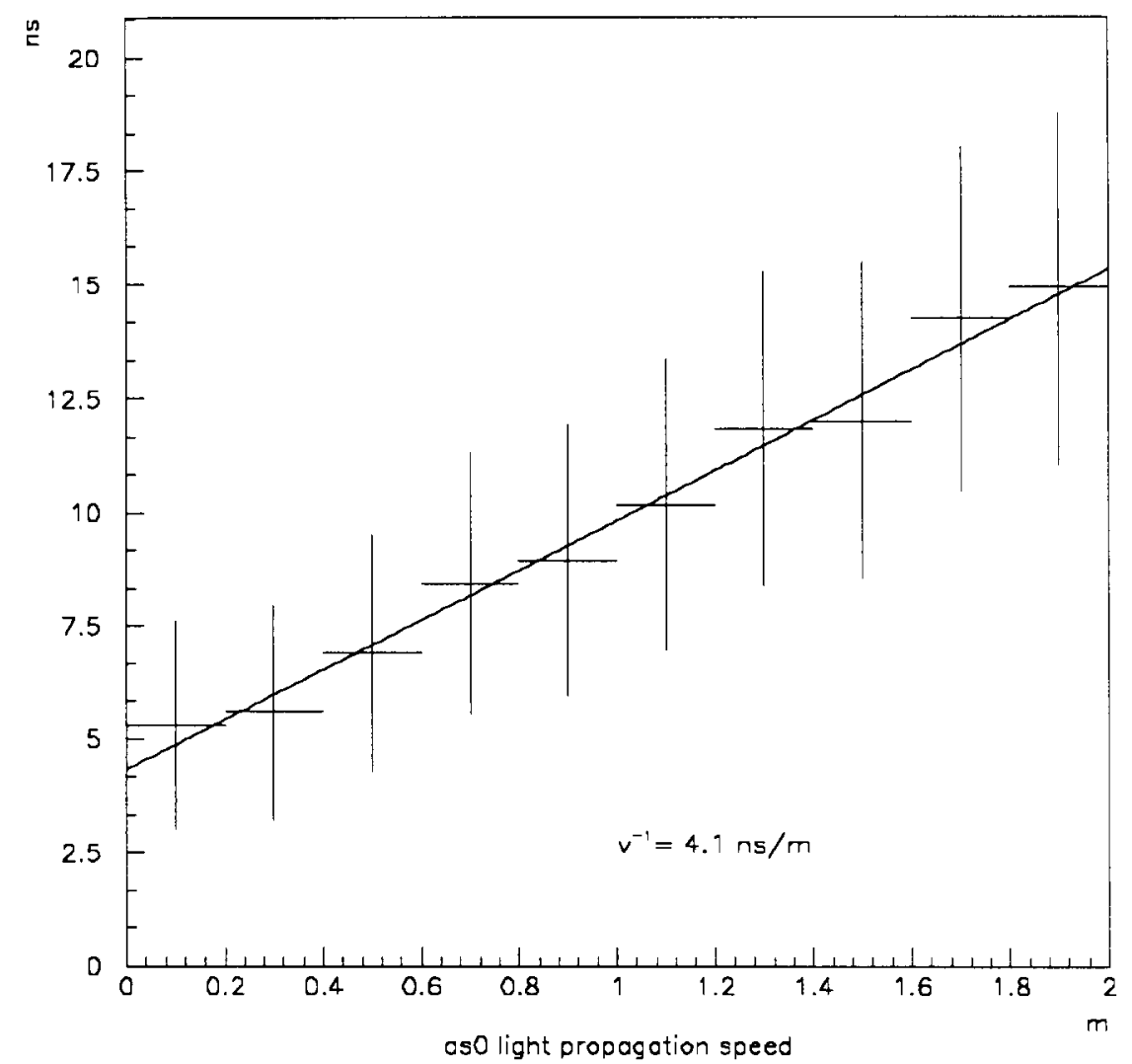

Fig. 15. Example of a measurement of light propagation speed in a fibre performed in the RIFOS experiment. 
On each fibre, the following measurements have been performed:

- average number of produced photoelectrons;

- attenuation length;

- time response;

- light propagation speed in the fibre.

\subsection{Attenuation length and average number of photoelectrons}

The attenuation length is computed by measuring the average number of photoelectrons produced by the photocathode of a photomultiplier placed at one end of the fibre, as a function of the source distance from the photocathode. If the source is far enough ( $>$ $0.5 \mathrm{~m}$ ) from the photocathode, the following formula may be used:

$N_{\mathrm{pe}}=N_{0} \exp \left(-x / \Lambda_{\text {att }}\right)$,

where $\Lambda_{\text {att }}$ is, by definition, the fibre attenuation length; $N_{0}$ is the number of photoelectrons extrapolated to zero distance. We extract the average number of photons produced by the fibre by dividing $N_{0}$ by the photocathode quantum efficiency for that scintillation light wavelength.

For this measurement, a $q V t$ module, used in $q$ mode and gated by the trigger system, receives the signal from the photomultiplier. Fig. 11 shows an example of a $q V t$ charge spectrum collected at a given position of the source along the fibre: the experimental distribution receives contributions from the noise (pedestal) and from the signals originated by $1,2, \ldots$ photoelectrons, smeared out by the statistical fluctuations in the multiplication process (we assume Gaussian distributions). Thus the $q V t$ histogram was fitted by the following expression:

$$
\begin{aligned}
f(x)= & \frac{w_{\text {ped }}}{\sqrt{2 \pi} \sigma_{\text {ped }}} \exp \left[-\frac{\left(x-x_{\text {ped }}\right)^{2}}{2 \sigma_{\text {ped }}^{2}}\right] \\
& +\sum_{l=1}^{M} \frac{w_{l}}{\sqrt{2 \pi} \sigma_{l}} \exp \left[-\frac{\left(x-\bar{x}_{l}\right)^{2}}{2 \sigma_{l}^{2}}\right] ;
\end{aligned}
$$

$M$ is the number of Gaussian photoelectron peaks contributing to the measured spectrum; $\bar{x}_{\text {ped }}$ and $\sigma_{\text {ped }}$ are the pedestal position and width respectively, $\bar{x}_{t}$ and $\sigma_{l}$ are the parameters of the $i$ th photoelectron contribution. We assume $\bar{x}_{t+1}-\bar{x}_{l}=$ constant and $\sigma_{l}=$ $\sqrt{i} \sigma_{1} ; \bar{x}_{\text {ped }}, \sigma_{\text {ped }}, \bar{x}_{1}, \sigma_{1}$ were determined in special runs with low light yield; $w_{l}, w_{\text {ped }}$ are the peak areas, to be determined through the fitting procedure. The peak areas $w_{t}$ reasonably match a Poisson distribution with mean value $\mu$ :

$w_{l}(t ; \mu)=K \frac{\mathrm{e}^{-\mu} \mu^{t}}{l !}$,

where $K$ is a normalization factor; $\mu$ is the average number of photoelectrons produced by the photocathode and it is obtained by a fit of the experimental parameters $w_{\imath}$ with the Poisson distribution (see fig. 12 as an example of this computation). We also tried a more complicated parameterization of the spectrum, allowing for Landau tails too: the differences in the results are not larger than $10 \%$; therefore we estimate the final uncertainty on the determination of $\mu$ to be $\pm 10 \%$. As a matter of fact we performed this procedure only at a few points of the attenuation curve to obtain absolute normalization: the attenuation curve was obtained simply by measuring the anode current as a function of the source position. An example of an attenuation plot is shown in fig. 13.

\subsection{Time response}

The time characteristic of a fibre is evaluated by using a LeCroy $q V t$ module in $t$ mode: the time behaviour of the scintillation pulse produced as the source is placed $5 \mathrm{~cm}$ away from the fibre end is measured. The start is given by the trigger system, and the stop by the first photon arriving at the photomultiplier. A high photon rate can lead to misleading results. For this reason, it is necessary to use filters in order to decrease the light yield seen by the photomultiplier to the level of a single photon per trigger, especially for blue scintillation light $(420-430 \mathrm{~nm})$. As foreseen by the theory [8], the scintillation pulse rises quite fast (it reaches its maximum value in about $1-2 \mathrm{~ns}$ ), it then decreases exponentially owing to the fluorescence decay time of the dye contained in the fibre; more precisely, the observed behaviour can be parameterized by the sum of several exponential functions, depending on the nature of dyes in the fibre. We estimate the electronic time resolution contributing to the time measurement to be $<0.6 \mathrm{~ns}$. An example of this measurement is shown in fig. 14 .

\subsection{Light propagatton speed}

The light propagation speed in each fiber is computed by moving the source along the fibre and performing 25 measurements of arrival time at each position. Moreover, we can measure the time spread of the arriving signals at each position: this time resolution is dominated by the fibre time response, and, together with the light propagation speed, it sets an ultimate limit to the "promptness" of the signals obtained by the fibre system. An example of these measurements is shown in fig. 15 .

\subsection{Photomultiplier quantum efficiency}

The quantum efficiency of the photomultipliers used in our tests was measured as follows (fig. 16). A 


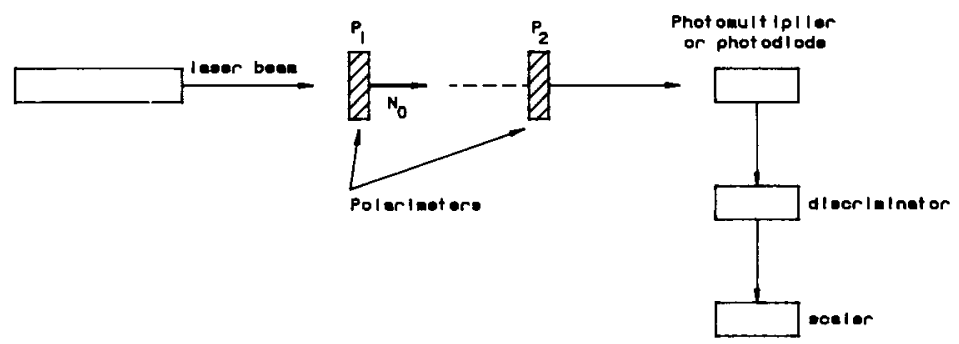

Fig. 16. Schematic representation of the experimental set-up used for measuring the quantum efficiency of the photomultiplier used in the RIFOS experiment.

laser beam crosses two polarimeters, before impinging onto either a photodiode or a photomultiplier; the axis of the first polarimeter was fixed, while the axis of the second one is rotated in order to increase or decrease the photon flux reaching the photon detector. If $N_{0}$ is the photon rate coming out of the first polarimeter, the number of photons per unit time reaching the photodiode is $N_{0}\left(\cos ^{2} \phi+k\right)$, where $\phi$ is the angle between the two polarimeter axes, and $k$ is a constant to be determined; we chose $\phi=\pi / 4$ so that the photon beam reaching the photodiode was intense enough for a reliable measurement of the current in the photodiode. If $\varepsilon_{\mathrm{p}}$ is the photodiode quantum efficiency $(60 \% \pm 5 \%)$, we get the photodiode current:
$I=e \varepsilon_{\mathrm{p}} N_{0}\left(\frac{1}{2}+k\right)$.

If $k$ is negligible (and in fact it is $\sim 10^{-7}$ ) we can extract the photon flux $N_{0}$.

Afterwards, the photodiode was replaced by a photomultiplier; the second polarimeter axis was rotated so that the final photon flux reached a minimum around $\phi=\pi / 2$, and the resulting photoelectron rate $N$ ( $\varepsilon_{\mathrm{pm}}$ is the photomultiplier quantum efficiency) was measured around the minimum value as a function of $\phi$ :

$N(\phi)=\varepsilon_{\mathrm{pm}} N_{0}\left(\cos ^{2} \phi+K\right)$.

$K$ is another experimental constant. The values of $\varepsilon_{\mathrm{pm}}$

Table 1

Experimental values for the main parameters of the scintillating fibres used for the RIFOS experiment

\begin{tabular}{|c|c|c|c|c|c|c|}
\hline \multirow{3}{*}{ No. $-\lambda_{\mathrm{em}}[\mathrm{nm}]$} & \multicolumn{6}{|c|}{ Fibre characteristics } \\
\hline & \multicolumn{3}{|c|}{ General } & \multirow{2}{*}{$\begin{array}{l}\text { Luminescence } \\
\tau[\mathrm{ns}]\end{array}$} & \multicolumn{2}{|l|}{ Propagation } \\
\hline & Diam. $[\mathrm{mm}]$ & $\Lambda_{\text {att }}[\mathrm{cm}]$ & $N_{0}$ & & $v^{-1}[\mathrm{~ns} / \mathrm{m}]$ & $\sigma[\mathrm{ns}]$ \\
\hline all -650 & 1 & 91 & $<0.3$ & $6.0 \pm 0.3$ & $6.2 \pm 1.1$ & $1.3 \pm 0.4$ \\
\hline al2 - 600 & 1 & 83 & $<0.3$ & $3.2 \pm 0.2$ & $6.0 \pm 0.9$ & $0.5 \pm 0.2$ \\
\hline al3 - 590 & 1 & 133 & 0.3 & $5.7 \pm 0.1$ & $6.1 \pm 0.1$ & $0.9 \pm 0.3$ \\
\hline al0 $-530 A$ & 1 & 368 & 0.9 & $15.9 \pm 0.2$ & $6.1 \pm 0.4$ & $2.9 \pm 0.5$ \\
\hline al5 -530 & 1 & 354 & 1.0 & $14.9 \pm 0.3$ & $6.0 \pm 1.8$ & $2.6 \pm 0.5$ \\
\hline al51 - 530/1 & 1 & 195 & 3.0 & $8.5 \pm 0.1$ & $5.8 \pm 0.5$ & $0.6 \pm 0.2$ \\
\hline al4 - 460 & 1 & 148 & 7.0 & $3.5 \pm 0.1$ & $6.6 \pm 0.4$ & $0.3 \pm 0.2$ \\
\hline al6 -430 & 1 & 174 & 6.0 & $2.3 \pm 0.1$ & $6.6 \pm 0.5$ & $0.3 \pm 0.2$ \\
\hline al7 -420 & 1 & 204 & 5.9 & $2.6 \pm 0.2$ & $6.6 \pm 0.5$ & $0.2 \pm 0.1$ \\
\hline al71-420A & 1 & 161 & 6.0 & $2.9 \pm 0.1$ & $6.5 \pm 0.5$ & $0.3 \pm 0.2$ \\
\hline $\mathrm{K} 3 \mathrm{HF}-530$ & 0.8 & 349 & 3.6 & $8.3 \pm 0.1$ & $6.5 \pm 1.3$ & $0.3 \pm 0.2$ \\
\hline as $1-650$ & 0.5 & 283 & 0.1 & $6.5 \pm 0.3$ & $5.8 \pm 0.5$ & $1.5 \pm 0.4$ \\
\hline as $2-600$ & 0.5 & 55 & 0.2 & $2.8 \pm 0.2$ & $5.8 \pm 0.5$ & $1.5 \pm 0.4$ \\
\hline as $3-590$ & 0.5 & 54 & 0.3 & $4.8 \pm 0.2$ & $6.5 \pm 0.5$ & $1.0 \pm 0.3$ \\
\hline as $0-530 \mathrm{~A}$ & 0.5 & 283 & 0.4 & $15.0 \pm 1$ & $5.5 \pm 1.7$ & $3.2 \pm 0.6$ \\
\hline as $51-530 / 1$ & 0.5 & 214 & 1.3 & $4.8 \pm 0.2$ & $6.6 \pm 0.5$ & $1.0 \pm 0.3$ \\
\hline as $52-530 / 2$ & 0.5 & 221 & 0.5 & $17.2 \pm 0.3$ & $5.9 \pm 1.6$ & $3.1 \pm 0.5$ \\
\hline as $4-460$ & 0.5 & 142 & 1.1 & $3.5 \pm 0.2$ & $6.4 \pm 1.2$ & $0.7 \pm 0.3$ \\
\hline as $6-430$ & 0.5 & 142 & 3.3 & $2.3 \pm 0.1$ & $6.9 \pm 0.5$ & $0.4 \pm 0.2$ \\
\hline as $7-420$ & 0.5 & 188 & 4.3 & $2.59 \pm 0.05$ & $6.6 \pm 0.5$ & $0.3 \pm 0.2$ \\
\hline as $71-420 \mathrm{~A}$ & 0.5 & 177 & 2.7 & $2.78 \pm 0.05$ & $6.7 \pm 0.5$ & $0.4 \pm 0.2$ \\
\hline
\end{tabular}


and $K$ are given by a fit of $N(\phi): \varepsilon_{\mathrm{pm}}$ is $5 \% \pm 1 \%$ for green light $(530 \mathrm{~nm})$ lasers, in agreement with the nominal value for a photomultiplier with a bialkali photocathode [7].

\subsection{Experimental results}

The experimental parameters for $1 \mathrm{~mm}, 0.8 \mathrm{~mm}$ and $0.5 \mathrm{~mm}$ diameter fibres are reported in table 1 . Column 1 shows the conventional abbreviation and the emission wavelength of each sample; column 2 shows the diameter of each fibre; columns 3 and 4 show the experimental values of $\Lambda_{\text {att }}$ and $N_{0}$, respectively. Column 5 shows the results of the measurements of the luminescence decay time of each fibre. Finally, column 6 shows the experimental values of the inverse of the propagation speed of light in each fibre, and column 7 shows the statistical standard deviation of $v^{-1}$. These results will be discussed in section 4 .

\section{Concluding remarks}

Looking at table 1 we see that several commercially available fibres have an attenuation length greater than $2.5 \mathrm{~m}$ (value taken as a guideline from the Monte Carlo simulation) even for $0.5 \mathrm{~mm}$ fibres. In particular, let us consider the as 51 , as 52 and as 0 , emitting green scintillation light $(530 \mathrm{~nm})$ : for these fibres, given a $P M$ quantum efficiency of $\simeq 5 \%$, the number of photons extrapolated to $0\left(N_{0}^{\gamma}\right)$ is $\simeq 15-20$, again in agreement with the Monte Carlo requirements.

From table 1 we see that the decay time for green fibres is generally longer than for blue fibres, but the spread of the arrival time is always $\lesssim 3$ ns. The inverse of light propagation speed is $\simeq 6 \mathrm{~ns} / \mathrm{m}$ for all the fibres; this speed, together with the arrival time spread, would limit the sensitive length of the fibres to $\simeq 2 \mathrm{~m}$ if the detector is to be ready at each bunchcrossing (every $15 \mathrm{~ns}$ ).

In conclusion, this investigation shows that the already commercially available fibres $(0.5-1 \mathrm{~mm})$ in diameter are almost adequate for a tracker detector at future hadron colliders. Also the radiation resistance of these fibres to the radiation doses expected at the LHC seems adequate [9].

On the other hand, due to the relatively low number of produced photons, a photon detector with high quantum efficiency ( $>50 \%$ ) and very low noise is necessary, and future efforts should be addressed mainly in this direction.

\section{Acknowledgements}

We would like to thank S. Lami for his contribution at the initial stage of the Monte Carlo calculation.

Also we warmly thank G. Stefanini for the continuous support and advice he has provided during this work.

M. Del Colletto, D. Rizzi and E. Pucciarelli gave a valuable technical support for the realization of this experiment.

\section{References}

[1] T. Mouthuy, ASCOT/EAGLE Internal Note, INDETNO-012 (1992).

[2] R. Brun et al., GEANT User's Guide, DD Service, CERN (1991).

[3] M. Atac and M.D. Petroff, Scintillating fiber tracking using visible light photon counters, in: Proc. Symp. on Detector R\&D for the SSC, Fort Worth, Texas, 1990.

[4] D. Denegri, Standard model physics at the LHC (pp collisions), in: Proc. of the Large Hadron Collider Workshop, Aachen, 1990, vol. I, eds. G. Jarlskog and D. Rein (CERN 90-10).

[5] ATLAS, Letter of Intent for a General Purpose pp Experiment at the Large Hadron Collider at CERN, CERN/LHCC/92-4,LHCC/12.

[6] See, for instance: D. Crosetto and L. Love, SSCL-576 (1992).

[7] Photomultiplier Tubes, Hamamatsu Photonics K.K., Electron Tube Division, 314-5, Shimokanzo, Toyookavillage, Iwata-gun, Shizuoka-ken, 438-01 Japan.

[8] J.M. Flournoy, An introduction to organic scintillators, in: Proc. Workshop on Scintillatıng Fiber Detector Development for the SSC, Fermilab, 1988.

[9] D. Bisello et al., Nucl. Instr. and Meth. A 325 (1993) 446. 